\section{Pleural sarcoidosis: a rare presentation}

\author{
E Loughney, B G Higgins
}

\begin{abstract}
Sarcoidosis is a chronic disorder of unknown aetiology which causes tissue injury and granuloma formation in many organs. Although over $80 \%$ of cases have intrathoracic disease at presentation, pleural sarcoidosis remains an unusual manifestation. A case of sarcoidosis presenting with a discrete pleural mass is reported. (Thorax 1997;52:200-201)
\end{abstract}

Keywords: sarcoidosis, pleural disease, mass lesion.

Sarcoidosis is a granulomatous disorder which may affect many organs throughout the body and therefore presents with a variety of symptoms and signs. Although pulmonary involvement is common, pleural disease is rare. We describe a case of sarcoidosis in which a discrete pleural mass was the presenting abnormality which predated the progression to parenchymal lung disease.

\section{Case report}

A 29 year old woman was admitted to hospital with sudden onset left sided pleuritic chest pain and two episodes of sputum streaked with haemoptysis. She gave a three week history of mild dyspnoea, malaise, and dry cough. Her current medications included norethisterone for irregular menstrual bleeding, and paroxetine, thioridazine, lithium carbonate, and procyclidine for bipolar psychosis. She smoked 10 cigarettes per day and consumed 15 units of alcohol per week.

On examination she was apyrexial and had no palpable lymphadenopathy or salivary gland enlargement. There was no evidence of joint disease, finger clubbing, skin rash, or eye disease. Respiratory examination revealed an area of dullness to percussion and reduced breath sounds posteriorly in the left mid zone. Cardiovascular, abdominal, and neurological examinations were normal. The chest radiograph showed a single, smooth, rounded homogeneous mass lesion in the left upper zone. There was mediastinal adenopathy but no evidence of hilar lymphadenopathy or parenchymal disease (fig 1). A ventilation perfusion scan showed a single matched defect at the area of radiological abnormality with a low probability of pulmonary embolus. Full blood count revealed a haemoglobin concentration of $11.9 \mathrm{~g} / \mathrm{dl}$, white blood cell count of $7.4 \times 10^{-9} \mathrm{~g} / \mathrm{dl}$ (granulocytes $75.9 \%$, lymphocytes $19.4 \%$, monocytes $4.7 \%$ ), and a platelet count of $202 \times 10^{9} \mathrm{~g} / \mathrm{dl}$. Serum electrolytes, including total calcium concentration, and liver function tests were normal. Serum angiotensin converting enzyme (ACE) activity was $26 \mathrm{IU} /$ 1 (range 21-54). Sputum was negative for acid fast bacilli (AFB), fungi, and other organisms. An intradermal tuberculin test (100000 units/ $\mathrm{ml}$ multiple puncture test) was grade 1 . Arterial blood gas tensions on admission were normal $\left(\mathrm{pH} 7.41, \mathrm{PCO}_{2} 4.1 \mathrm{kPa}, \mathrm{Po}_{2} 13.7 \mathrm{kPa}\right) . \mathrm{Pul}-$ monary function tests showed forced expiratory volume in one second $\left(\mathrm{FEV}_{1}\right) 3.451$ (predicted 3.101 ), vital capacity (VC) 4.201 (predicted 3.601 ), total lung capacity (TLC) 6.851 (predicted 4.95 l), residual volume (RV) 2.651 (predicted $1.45 \mathrm{l}$ ), carbon monoxide transfer factor (TLCO) $6.23 \mathrm{mmol} / \mathrm{min} / \mathrm{kPa}$ (predicted 8.42 ), and transfer coefficient (KCO) of $1.38 \mathrm{mmol} /$ $\min / \mathrm{kPa} / 1$ (predicted 1.79). A computed tomographic (CT) scan of the thorax revealed a pleural based mass in the left mid zone with no involvement of bone but an associated lymphadenopathy affecting the superior mediastinal, hilar, and subcarinal stations.

Biopsy specimens were obtained from the pleural mass and mediastinal nodes. Histological examination of the nodes revealed multiple non-caseating granulomas composed of epithelioid cells and multinucleated Langhans giant cells with surrounding irregular lymphocytic infiltration. The pleural mass was composed of fibrous tissue housing non-caseating granulomas. At both sites the appearances were consistent with a diagnosis of sarcoidosis.

Symptoms settled with conservative treatment and the patient remained asymptomatic for 12 months. She then developed increasing breathlessness with an associated change in pulmonary function $\left(\mathrm{FEV}_{1} 2.85 \mathrm{l}\right.$, VC 3.551 , TLco $4.04 \mathrm{mmol} / \mathrm{min} / \mathrm{kPa}$, Kco $1.03 \mathrm{mmol} /$ $\min / \mathrm{kPa} / \mathrm{l})$. Diffuse nodular shadowing developed throughout both lung fields on the chest radiograph. Prednisolone was started at a dose of $30 \mathrm{mg} /$ day, leading to improvement in pulmonary function $\left(\mathrm{FEV}_{1} 3.25 \mathrm{l}\right.$, VC 3.951 , TLCO $5.20 \mathrm{mmol} / \mathrm{min} / \mathrm{kPa}$ and $\mathrm{KcO} 1.30 \mathrm{mmol} /$ $\min / \mathrm{kPa} / \mathrm{l})$ and partial regression of the nodular shadowing after several weeks of treatment. There was a steady decline in the size of the pleural mass over the following nine months with continued clinical improvement.

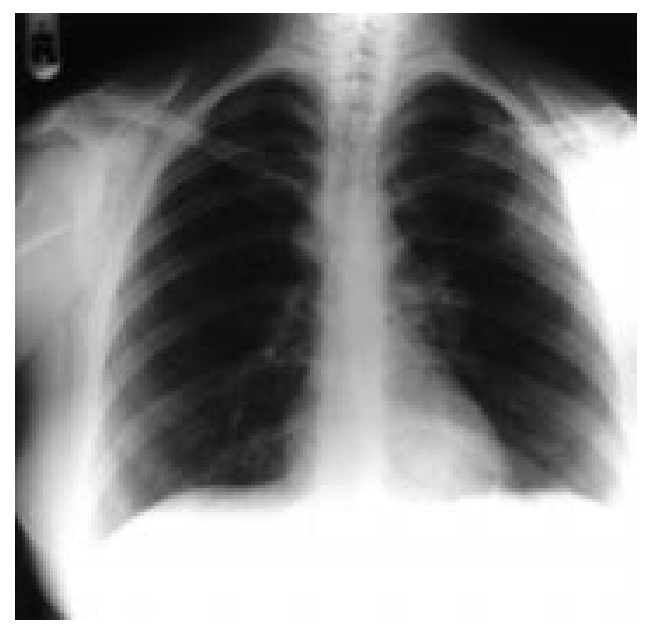

Figure 1 Chest radiograph showing pleural mass at presentation. 


\section{Discussion}

Pleural thickening and abnormalities of the subpleural region have been recognised increasingly in sarcoidosis by use of high resolution CT scanning. ${ }^{1}$ However, a pleural mass has not previously been reported.

Pleural manifestations of sarcoidosis are generally rare; in two recent reviews of sarcoidosis pleural disease was present in $0.16 \%^{2}$ and $0.73 \%{ }^{3}$ of cases. The diagnosis of pleural sarcoidosis is usually based on the presence of pleural effusions or thickening, ${ }^{4}$ but historically histological confirmation has not always been available. Effusions may be unilateral or bilateral, transudates or exudates, and may or may not be associated with parenchymal disease. ${ }^{56}$ Pleural manifestations may arise at initial presentation, which appears to be the situation in our case since the disease later progressed, or at a later stage in the development of known thoracic sarcoidosis.

The development of pleural sarcoidosis does not appear to have any clear prognostic value. Some reports suggest a benign course ${ }^{1}$ but relentless deterioration with development of parenchymal disease has also been described. ${ }^{2}$ Involvement of the pleura in sarcoidosis has been reported in association with disease of the liver, skin, and joints. ${ }^{7}$
The cause of breathlessness in our patient remains unknown. However, thoracic lymphadenopathy and concomitant compression of the pulmonary arterial circulation was thought to have led to breathlessness in two previously reported cases of sarcoidosis. ${ }^{89}$ Interestingly, in each of these cases the subject was female and was receiving oral progestogen therapy as in our case; treatment with prednisolone reportedly led to the amelioration of breathlessness on each occasion.

1 Batra P. Role of high-resolution CT in the diagnosis and evaluation of pulmonary sarcoidosis. Sarcoidosis 1993;10: 957

2 Tommasini A, Di Vittorio G, Fachinetti F, Festi G, Schito V, Cipriani A. Pleural effusion in sarcoidosis: a case report. Sarcoidosis 1994;11:138-40.

3 Salzar A, Mana J, Corbella X, Vidaller A. Sarcoid pleural effusion: a report of two cases. Sarcoidosis 1994:11:1357.

4 Beekman JF, Zimmet SM, Chun BK, Miranda AA, Katz S. Spectrum of pleural involvement in sarcoidosis. Arch Intern Med 1976;136:323-30.

5 Durand DV, Dellinger A, Guerin C, Guerin JC, Levrat R. Pleural sarcoidosis: one case presenting with an eosinophilic effusion. Thorax 1984;39:468-9.

6 Kanada DJ, Scott D, Sharma OP. Unusual presentations of pleural sarcoidosis. Br f Dis Chest 1980;74:203-5.

7 Nelson DG, Louden RG. Sarcoidosis with pleural involvement. Am Rev Respir Dis 1973;108:647-51

8 Finestone H, Colp C, Rackson M, Shams J, Gallagher R. Ventilation-perfusion imaging in sarcoidosis:potential for nonembolic segmental mismatch. F Nucl Med 1994;35 476-8.

9 Goffman TE, Bloom RL, Dvorak VC. Acute dyspnoea in a young woman taking birth control pills. $\mathcal{F} A M A$ 1984;251: $146-56$.

Thorax 1997;52:201-202

\section{Sarcoidosis and primary biliary cirrhosis with co-existing myositis}

\author{
P Hughes, C R McGavin
}

\begin{abstract}
In a small number of cases the co-existence of primary biliary cirrhosis and sarcoidosis is assumed from clinical serological and histological findings. A case of sarcoidosis is reported in which the $\mathrm{M} 2$ antibody, a highly specific marker for primary biliary cirrhosis, was detected. The patient also developed a severe myositis and a possible overlap syndrome is discussed. (Thorax 1997;52:201-202)
\end{abstract}

Keywords: sarcoidosis, primary biliary cirrhosis, overlap syndrome.

Department of Respiratory Medicine, Freedom Fields Hospital, Plymouth Devon PL4 7JJ, UK P Hughes

C R McGavin

Correspondence to: Dr C R McGavin.

Received 26 May 1995 Returned to authors 22 September 1995

Revised version received

4 December 1995

Accepted for publication

8 December 1995
Hepatic granulomas in sarcoidosis are rarely of clinical significance, but occasionally a cholestatic picture occurs which has marked similarities to primary biliary cirrhosis. ${ }^{1}$ In a few reported cases there is evidence to suggest the co-existence of these two disorders, prompting suggestions of an overlap syndrome. ${ }^{2-4}$

We present a case of sarcoidosis with granulomatous hepatitis and immunological features of primary biliary cirrhosis in which a florid polymyositis developed. This feature has not been noted in previous cases of overlap.
Case report

A 48 year old white woman presented in 1980 with features of sarcoidosis affecting her skin, nasal mucosa, lungs, and lachrymal and parotid glands. Characteristic non-caseating granulomas were seen on biopsy specimens of skin and bronchial mucosa.

Investigations revealed a moderate eosinophilia $\left(2.34 \times 10^{9} / 1\right)$, mild liver function abnormality (alkaline phosphatase (ALP) 55, gamma-glutamyl transferase (GGT) 158) and negative autoantibody screen except antimitochondrial antibody (AMA) positive at 1: 40. The chest radiograph and pulmonary function tests confirmed significant lung involvement and prednisolone $20 \mathrm{mg}$ daily was started, tapering to $5 \mathrm{mg}$ daily. There was a very satisfactory clinical and physiological response.

After a period of clinical stability the patient re-presented in 1984 with lethargy, myalgia, and leg weakness. Investigations showed a creatinine kinase (CK) level of 1896 IU/1 (24-170) with electromyographic evidence of myositis. A muscle biopsy revealed chronic inflammation and necrosis without granulomas, eosinophils, or vasculitis (fig 1). During these investigations a deterioration in liver function was noted (ALP 230, GGT 170) with strongly positive AMA at 1:1560. A liver biopsy specimen showed illdefined granulomas, histologically compatible with sarcoidosis (fig 2).

The myositis was controlled over the next six years with prednisolone $20 \mathrm{mg}$ daily during which time biochemical evidence of anterior pituitary failure emerged. Further hepatic deterioration occurred but without pruritus or jaundice. In 1990 investigations showed ALP 


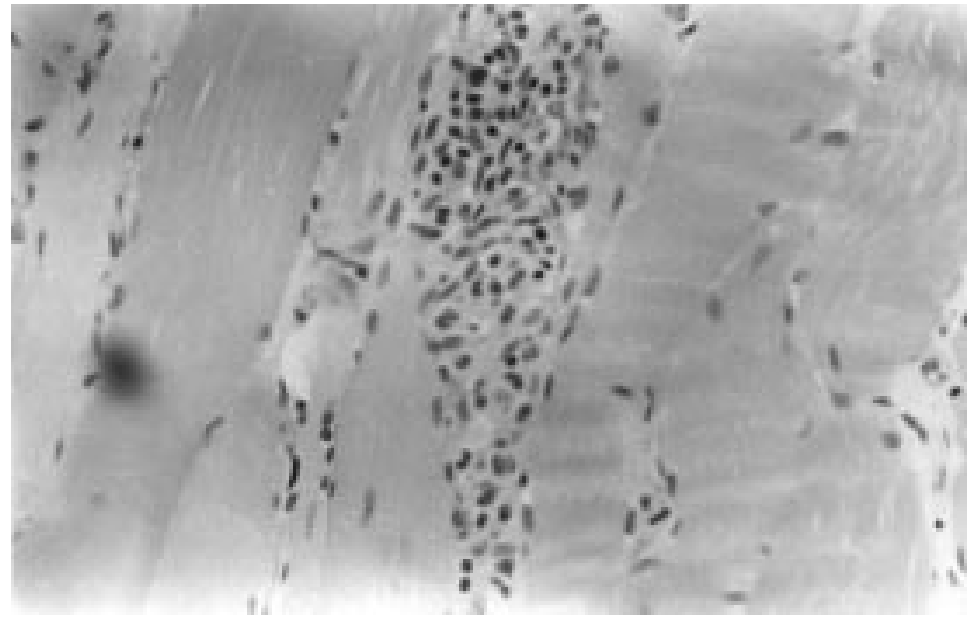

Figure 1 Photomicrograph of muscle biopsy tissue showing non-eosinophilic inflammatory changes with necrosis. Original magnification $\times 400$.

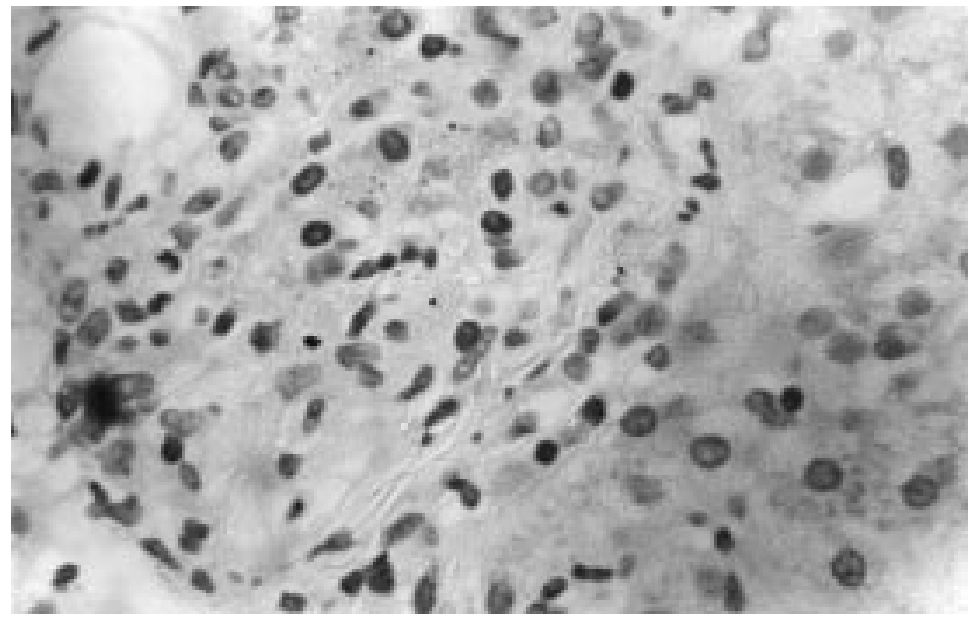

Figure 2 Photomicrograph of liver biopsy tissue showing non-caseating granulomas. Original magnification $\times 630$.

274, GGT 400, AMA 1:2560, IgG 9.24 (0.5-14) g/l, IgM $5.1(0.5-2) \mathrm{g} / 1$, cholesterol $7.5 \mathrm{mmol} / 1$, CK $34000 \mathrm{IU} / 1$. Tests for antineutrophil cytoplasmic antibody and extractable nuclear antigen were negative. Biopsy samples of liver and muscle showed no significant change. She continued to be managed with prednisolone $10 \mathrm{mg}$ daily but was disabled by myalgia and proximal weakness.

In 1992 worsening myositis (CK 5148 IU/1) with further decline in liver function (ALP 420, GGT 1132) required the addition of cyclophosphamide, with dramatic improvement in symptoms and biochemistry (CK 134 IU/l, ALP 151, GGT 455). The AMA remained positive. The cyclophosphamide was initially given in pulsed $750 \mathrm{mg}$ doses, later reduced to $50 \mathrm{mg}$ on alternate days. In 1993 a strongly positive mitochondrial M2 EIA was also noted.

\section{Discussion}

In 1969 Karlish et al reported a patient with cholestasis, mediastinal lymphadenopathy, and positive Kveim and AMA tests. There has followed a small number of cases ${ }^{34}$ where the co- existence of sarcoidosis and primary biliary cirrhosis has been suggested, usually by demonstrating the AMA in the presence of either a positive Kveim test or, as in our case, multiorgan granulomas. An increased IgM often adds further weight to the diagnosis of primary biliary cirrhosis. Although the AMA test is positive in $85-99 \%$ of cases of primary biliary cirrhosis, it is present in $0.7 \%$ of controls as well as other autoimmune and chronic hepatic disorders; its significance in these cases is therefore unclear. ${ }^{5}$ More recently the M2 mitochondrial antibody directed at components of the pyruvate dehydrogenase complex on the inner mitochondrial membrane has been shown to be more highly specific for primary biliary cirrhosis, ${ }^{6}$ and has not previously been reported in sarcoidosis.

These cases of co-existing primary biliary cirrhosis and sarcoidosis, together with the realisation that primary biliary cirrhosis can affect the lung in a similar way to sarcoidosis, ${ }^{7}$ prompt suggestion of an overlap syndrome that may be of relevance to the myositis noted in our case.

In patients with sarcoidosis muscle granulomas, like those in the liver, are rarely of clinical significance and correlate poorly with symptoms. They are reported in all cases of sarcoid myositis (itself very rare) and are suggested as a distinguishing feature between polymyositis and the myositis of sarcoidosis. ${ }^{8}$ Primary biliary cirrhosis frequently overlaps with other autoimmune diseases including those with pulmonary involvement. Cases of polymyositis with primary biliary cirrhosis and a case of polymyositis with pulmonary fibrosis which later developed sarcoid granulomas are also described. ${ }^{7910}$

Although the co-existence of sarcoidosis and primary biliary cirrhosis is rare and granulomatous myositis is an infrequent occurrence in sarcoidosis, we have not seen granulomas in either muscle biopsy specimen and therefore conclude that our case is an overlap between sarcoidosis, primary biliary cirrhosis, and polymyositis.

The authors thank the many clinicians and pathologists who have contributed to the diagnosis and management of this case and Angela Parker for secretarial assistance.

1 Maddrey WC, Johns CJ, Boitnott JK, Iber FL. Sarcoidosis and chronic hepatic disease. Medicine 1970;49:375-95.

2 Karlish AJ, Thompson RPH, Williams R. A case of sarcoidosis and primary biliary cirrhosis. Lancet 1969;ii:599.

3 Fagan EA, Moore-Gillon JC, Turner-Warwick M. Multiorgan granuolomas and mitochondrial antibodies. $N$ Engl f Med 1983;308:572-80.

4 Stanley NM, Fox RA, Whimster WF, Sherlock S, James DG. Primary biliary cirrhosis or sarcoidosis or both? $N$ Engl f Med 1972;287:1282-4.

5 Klatskin G, Kantor FS. Mitochondrial antibody in primary biliary cirrhosis and other disease. Ann Intern Med 1972; 77:533-41

6 Yeaman SJ, Danner DJ, Mutimer DJ, Fussey SPM, James OFW, Bassendine MF. Primary biliary cirrhosis: identification of two major M2 mitochondrial autoantigens. tification of two major M2 mitochondrial autoantigens.
Lancet 1988;i:1067-70.

7 Wallace JG, Tong MJ, Veki Bh, Quismorio FP. Pulmonary involvement in primary biliary cirrhosis. $\mathcal{F}$ Clin Gastroenterol 1987;9:431-5.

8 Ando DG, Lynch JP, Faentone JC. Sarcoid myopathy with elevated creatinine phosphokinase. Am Rev Respir Dis 1985;131:298-300.

9 Milosovic M, Adams P. Primary biliary cirrhosis and polymyositis. F Clin Gastroenterol 1990;12:332-5.

10 Callen JP. Sarcoidosis appearing initially as polymyositis. Arch Dermatol 1979;115:1336-7. 